\title{
External optical self-injection stabilization of an InP generic foundry platform based passively mode- locked ring laser
}

Auth, Dominik, Weber, Christoph, Lo, Mu-Chieh, Fiala, Patrick, Sauer, Pascal, et al.

Dominik Auth, Christoph Weber, Mu-Chieh Lo, Patrick Fiala, Pascal Sauer, Guillermo Carpintero, Stefan Breuer, "External optical self-injection stabilization of an InP generic foundry platform based passively mode-locked ring laser," Proc. SPIE 11301, Novel In-Plane Semiconductor Lasers XIX, 113010V (24 February 2020); doi: 10.1117/12.2547146 


\title{
External optical self-injection stabilization of an InP generic foundry platform based passively mode-locked ring laser
}

\author{
Dominik Auth ${ }^{\mathrm{a}}$, Christoph Weber ${ }^{\mathrm{a}}, \mathrm{Mu}-\mathrm{Chieh} \mathrm{Lo}^{\mathrm{b}, \mathrm{c}}$, Patrick Fiala ${ }^{\mathrm{a}}$, Pascal Sauer ${ }^{\mathrm{a}}$, Guillermo \\ Carpintero $^{\mathrm{c}}$, and Stefan Breuer ${ }^{\mathrm{a}}$ \\ anstitute of Applied Physics, Technische Universität Darmstadt, 64289 Darmstadt, Germany \\ ${ }^{\mathrm{b}}$ University College London, London WC1E 7JE, UK \\ ${ }^{c}$ Universidad Carlos III de Madrid, Av de la Universidad, 30. 28911 Leganés, Madrid, Spain
}

\begin{abstract}
Timing stabilization of a photonic integrated circuit extended cavity passively mode-locked semiconductor ring laser with four gain sections and two saturable absorbers in a symmetric ring geometry by optical self-injection is presented. The laser has been fabricated using an InP generic integration technology platform. Repetition rate tuning up to $5.5 \mathrm{MHz}$ and a timing jitter reduction by optical self-injection from $99 \mathrm{fs}$ (solitary laser operation) to $20 \mathrm{fs}$ is demonstrated. The experimental results are in excellent agreement with results obtained by a stochastic time domain model which yet had been solely applied to edge-emitting straight waveguide semiconductor lasers.
\end{abstract}

Keywords: semiconductor laser, ring mode-locked laser, photonic integrated circuit, optical self-injection

\section{INTRODUCTION}

Photonic integrated circuit passively mode-locked lasers are small-footprint optical frequency comb sources which feature individual designs and are affordable due to the concept of multi-project wafer runs. ${ }^{1}$ InP based photonic integrated circuits offering central emission wavelengths in the C- and L-band are envisioned to play an important role in future communication systems. ${ }^{2}$ On-chip generation of one or multiple optical frequency combs enables use in spectroscopic experiments and remote distance sensing with minimized footprints. ${ }^{3-5}$ One way to realize passively mode-locked lasers on photonic integrated circuits using the standard building blocks of an openaccess multi-project-waver run foundry are extended cavity ring layouts. ${ }^{4,6-10}$ Favored by the effect of colliding pulse mode-locking within the saturable absorber region within the ring lasers ${ }^{11}$ and missing optical isolator building blocks, generally optical pulse trains in clockwise and counterclockwise direction within the resonator are observed simultaneously ${ }^{4,6-9}$ but efforts are made to achieve unidirectional operation. ${ }^{10}$

To control the pulse repetition rate of monolithic mode-locked lasers and improve the pulse timing stability optical self-injection has been studied first for quantum-well lasers in $1993^{12}$ and since then for mode-locked lasers with active regions based on quantum-well, ${ }^{13,14}$ quantum-dash ${ }^{15,16}$ and quantum-dot ${ }^{17-21}$ materials. To reproduce and predict the impact of optical self-injection by one or more external cavities a simple stochastic time-domain model has been developed ${ }^{19,22}$ and verified for passively and self mode-locked lasers with active regions based on quantum-dots ${ }^{19,23}$ and quantum-wells. ${ }^{24}$ The model is based on interaction between the backinjected optical pulses and the pulse within the laser cavity. By changing the length of the external self-injection cavity, a sawtooth shaped dependence of the pulse repetition rate in dependence on this length is expected. The maximum repetition rate control range is inversely depending on the total external self-injection cavity length. ${ }^{24}$ On the other hand, stronger timing stability improvement is expected for longer external cavities. ${ }^{19,22}$ This model hasn't yet been applied to simulate the impact of optical self-injection on photonic integrated circuit based lasers or to simulate extended cavity ring semiconductor lasers. Injecting back the optical pulse train from one propagation direction into the same waveguide leads to injection into the counter-propagating direction in the ring resonator.

Further author information: (Send correspondence to Stefan Breuer or Dominik Auth)

Stefan Breuer: E-mail: stefan.breuer@physik.tu-darmstadt.de,

Dominik Auth: E-mail: dominik.auth@physik.tu-darmstadt.de

\footnotetext{
Novel In-Plane Semiconductor Lasers XIX, edited by Alexey A. Belyanin,

Peter M. Smowton, Proc. of SPIE Vol. 11301, 113010V · @ 2020 SPIE CCC code: $0277-786 X / 20 / \$ 21 \cdot$ doi: $10.1117 / 12.2547146$
} 
In this conference proceeding, timing stabilization and repetition rate tuning of an InP photonic integrated circuit extended cavity passively mode-locked semiconductor ring laser by optical self-injection is demonstrated for the first time. Repetition rate tuning up to $5.5 \mathrm{MHz}$ and a timing jitter reduction from $99 \mathrm{fs}$ in solitary laser operation to $20 \mathrm{fs}$ with optical self-injection is demonstrated. The ring structure subject to optical selfinjection exhibits the same behaviour in timing jitter reduction and repetition rate tunability as Fabry-Perot lasers featuring the same cavity roundtrip time, indicating a similar intra-cavity pulse interaction of the backinjected pulses with the original pulses. Simulations by a stochastic model aim at supporting the experimental findings.

\section{DEVICE AND EXPERIMENTAL SET-UP}

The InP photonic integrated circuit has been built with a predefined layer stack with active regions based on InGaAsP/InP quantum-well gain media ${ }^{25}$ and features a symmetric extended cavity ring laser design, consisting of two saturable absorber section, four gain sections, four electro-optic phase modulator sections. Four optical outputs which are tilted by $7^{\circ}$ and are anti-reflective coated to minimize unwanted back-reflections. The design and optical self-injection set-up is schematically depicted in Fig. 1 and its solitary mode-locking properties have recently been investigated and reported. ${ }^{8}$ The laser fabrication was part of a multi-project wafer run through the Jeppix foundry coordinator at SMART Photonics. In this conference proceeding, the gain sections of the laser are injected with a total current of $240 \mathrm{~mA}$ while a reverse bias voltage of $-2.8 \mathrm{~V}$ is applied to the absorber sections. This operation parameters are chosen to achieve a broad pulse width of $2.2 \mathrm{ps}$ yielding a wide interaction width for optical self-injection and to ensure a high fiber-coupled output power of $0.2 \mathrm{~mW}$. The electro-optic phasemodulator sections and the booster gain section, located at output 3, are left unbiased. Using a lensed optical single-mode fiber the light emitted from output 4, which corresponds to clockwise propagating pulses within the ring-cavity, is coupled with an approximated efficiency of $30 \%$. By a fiber-based beam-splitter $50 \%$ of this light is sent to the optical self-injection cavity, consisting of an optical single-mode fiber embedding fiber-based polarization control, to optimize back-injection polarization, and a free-space based part consisting of a variable optical attenuator to control the back-injected optical power and a mirror mounted on a linear translation stage to control and tune the length of the optical self-injection cavity by $\mathrm{L}_{m i c}=9 \mathrm{~mm}$ corresponding to an additional round-trip time for the pulse of $60 \mathrm{ps}$.

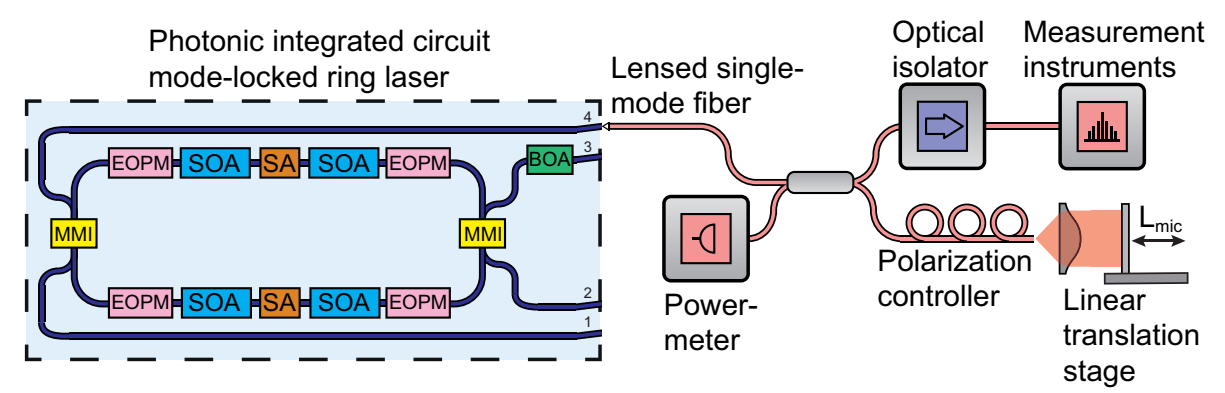

Figure 1. Schematic of the passively mode-locked extended cavity photonic integrated circuit ring laser layout and the experimental optical self-injection set-up. The symmetric cavity includes four gain sections (SOA) and two saturable absorber sections (SA). Two multimode-interference coupler (MMI) provide in total 4 outputs labeled 1 to 4 , to analyze light from the clockwise and the counter-clockwise propagation direction. The electro-optic phase modulators (EOPM) and the booster amplifier (BOA) in front of output 3 are left unbiased in the presented investigations. A lensed optical single-mode fiber is used for coupling the light from output 4. Optical self-injection is realized by a fiber-based self-injection cavity with a free-space part, consisting of a mirror mounted on a high-precision linear translation stage.

The other $50 \%$ of the light pass a fiber-based optical isolator (isolation $>28 \mathrm{~dB}$ ) to prevent residual optical feedback and directed towards the measurement equipment. Laser output power and mode-locking characteristics are studied by an optical spectrum analyzer (spectral resolution $10 \mathrm{pm}$ ), a fast photo-diode (electrical bandwidth $>45 \mathrm{GHz}$ ) in combination with an electrical spectrum analyzer (electrical bandwidth $>50 \mathrm{GHz}$ ) and an optical power meter. At the second input of the fiber-based beam-splitter, a power meter monitors the back-injected power sent back to output 4 of the photonic integrated circuit extended cavity symmetric passively 
mode-locked ring laser. The meausured emission characteristics in solitary operation are depicted in Fig. 2. The passively mode-locked laser emits $2.2 \mathrm{ps}$ short optical pulses, obtained by deconvoluting a well-matching Gaussian fit to the autocorrelation trace (autocorrelation trace and fit depicted in Fig. 2(d)) with a pulse repetition rate of $\mathrm{RR}_{0}=23.32 \mathrm{GHz}$ (radio-frequency spectrum depicted in Fig. 2(a)) corresponding to the single-roundtrip time of $\mathrm{T}_{0}=43 \mathrm{ps}$ of the optical ring cavity. Fundamental mode-locking is present at this laser biasing conditions. The free-running timing jitter amounts to $76 \mathrm{fs}$ and is obtained from the radio-frequency linewidth of the repetition rate ${ }^{26}$ determined from the $1 / \mathrm{f}^{2}$ dependency of the timing-phase-noise power-spectral density (TPN PSD), as depicted in Fig. 2(b). The optical frequency comb $(-3 \mathrm{~dB}$-width: $6.2 \mathrm{~nm})$ is centered at the L-band wavelength of $1571 \mathrm{~nm}$ (optical spectrum depicted in Fig. 2(c)). Due to the symmetric cavity design, unidirectional emission is not expected and clockwise and counter-clockwise propagating light within the cavity are confirmed. ${ }^{8}$ The fixed macroscopic optical path length of the optical self-injection cavity amounts to $16 \mathrm{~m}$ (roundtrip time $\mathrm{T}_{1}=2439 \mathrm{~T}_{0}$ ), the optical self-injection power is $12 \mu \mathrm{W}$.
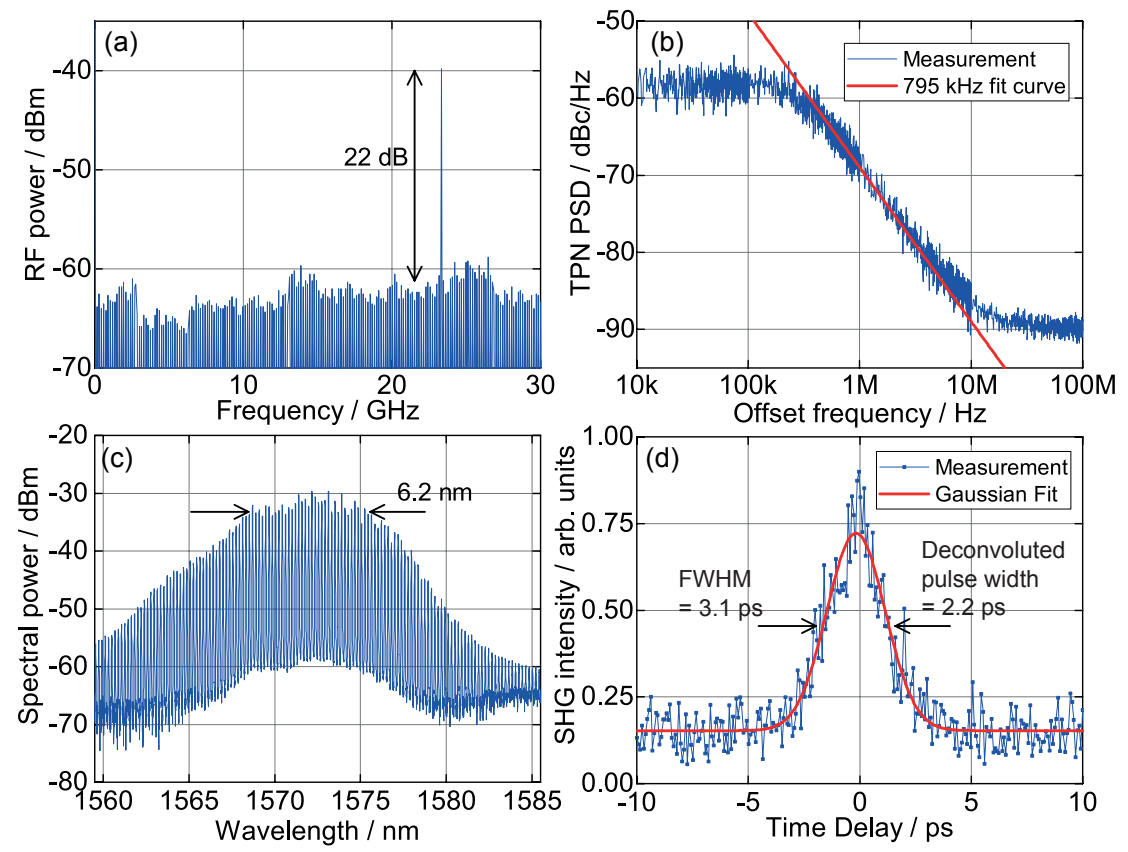

Figure 2. Solitary mode-locked laser results for a gain current of $240 \mathrm{~mA}$ and an absorber reverse bias voltage of $2.8 \mathrm{~V}$. (a) $\mathrm{RF}$ spectrum showing the repetition rate at $\mathrm{RR}_{0}=23.32 \mathrm{GHz}$, (b) TPN PSD of the repetition rate line illustrated with a $1 / f^{2}$ fit curve of $795 \mathrm{kHz}$ resulting in a pulse-to-pulse timing jitter of $99 \mathrm{fs}$, (c) Optical spectrum with a $-3 \mathrm{~dB}$ width of $6.2 \mathrm{~nm}$, (d) Auto-correlation time trace with a de-convoluted pulse width of $2.2 \mathrm{ps}$.

\section{STOCHASTIC TIME-DOMAIN MODEL}

To validate the experimentally studied dependencies of the repetition rate on the fine-delays, a stochastic time domain model is used which considers a passively mode-locked semiconductor laser as a free-running oscillator exhibiting timing deviations within each pulse round trip. ${ }^{19}$ One of the main noise source causing these deviations is amplified spontaneous emission directly coupling to the laser pulse and, thus, inducing repetition rate instabilities. This noise is uncorrelated and therefore white in frequency. After each round-trip, the timing deviation is accumulated leading to a Wiener process or a random walk. ${ }^{22}$ The timing deviation $\mathrm{T}(\mathrm{n}+1)$ from an ideal clock of the $\mathrm{n}+1$ pulse for optical self-feedback follows

$$
T(n+1)=\frac{T(n)+\sigma_{\mathrm{ptp}} \Gamma(n)+\gamma W(\Delta T) T\left(n-n_{d}\right)}{1+\gamma W(\Delta T)}
$$

where $\sigma_{\mathrm{ptp}}$ is the pulse-to-pulse timing jitter which is accessed by measuring the fundamental repetition rate linewidth in the RF spectrum with $\sigma_{\text {ptp }}=\left(\Delta \nu_{0} / 2 \pi \nu_{0}^{3}\right)^{0.5}$ where $\Delta \nu_{0}$ is the $-3 \mathrm{~dB}$ repetition rate line width 
and $\nu_{0}$ the repetition rate frequency, ${ }^{26} \gamma$ is the timing interaction strength directly correlated to the optical self-feedback strength in the experiment and $n_{d}$ is the discretized time delay of the optical self-feedback in terms of number of pulses. $\Gamma(n)$ is a Gaussian distributed random number with a standard deviation of 1 . The term $W(\Delta T)=W\left(T(n)-T\left(n-n_{d}\right)\right)$ is a weighting pulse interaction function correlated to the optical pulse width and is given in this work for the specified operation point by 3.1 ps (see Fig. 2(d)).

\section{RESULTS AND DISCUSSION}

Experimentally, the influence of optical self-injection with fine-delay cavity length tuning with nanometric precision onto the pulse repetition rate and the timing jitter of the emitted pulse train of the symmetric ring mode-locked laser is investigated. The pulses propagating in clockwise direction in the internal cavity are back injected into the same output and therefore their propagation direction changes and the back-injected pulses are propagating in counter-clockwise direction. By modelling with the stochastic time-domain model similarities to straight waveguide monolithic quantum-well mode-locked lasers are researched. The model is taken from ${ }^{19}$ and the simulation parameters are adapted to the experiment in this work, the interaction function $W(\Delta T)$ is given by $3.1 \mathrm{ps}$ for the specified operation point beeing the FWHM of the Gaussian fit to the auto-correlation trace depicted in Fig. 2(d). By moving the mirror mounted on the high precision stage the length of the optical self-injection cavity is elongated. The experimental repetition rate deviation from the solitary repetition rate $\left(\mathrm{RR}-\mathrm{RR}_{0}\right)$ as a function of the additional fine-delay is depicted in Fig. 3(a) in blue. In Fig. 3(b) the corresponding timing jitter, obtained by $\mathrm{f}^{2}$-fits to the TPN PSD spectra of the Lorentzian shaped repetition rate lines in the radio frequency spectra, ${ }^{26}$ as a function of the same fine-delay is depicted. The repetition rate deviation is found to be always below or equal 0 , the repetition rate influenced by optical self-injection is therefore lower than or equal to the solitary repetition rate for all fine-delays. The repetition rate can be continuous controlled in a range of $5.5 \mathrm{MHz}$ and the repetition rate deviation as a function of fine-delay shows a periodicity of $43 \mathrm{ps}$, corresponding to the internal laser cavity roundtrip time $T_{0}=43 \mathrm{ps}$. Within one of these periods, a plateau of non-changing repetition rate is evident, spanning $20 \mathrm{ps}$. For higher fine-delay values the repetition rate decreases with increasing fine-delay at an average of $-248 \mathrm{kHz} / \mathrm{ps}$. This shape indicates a limited locking-range between the back-injected and the intra-cavity pulses due to a too short pulse width and is in excellent agreement with results obtained for straight waveguide monolithic passively mode-locked quantum-well lasers. ${ }^{24}$ Due to this limited interaction the maximum repetition rate tuning range of approximately $9 \mathrm{MHz}$ for the investigated optical self-injection cavity length of $16 \mathrm{~m}$ is not reached.
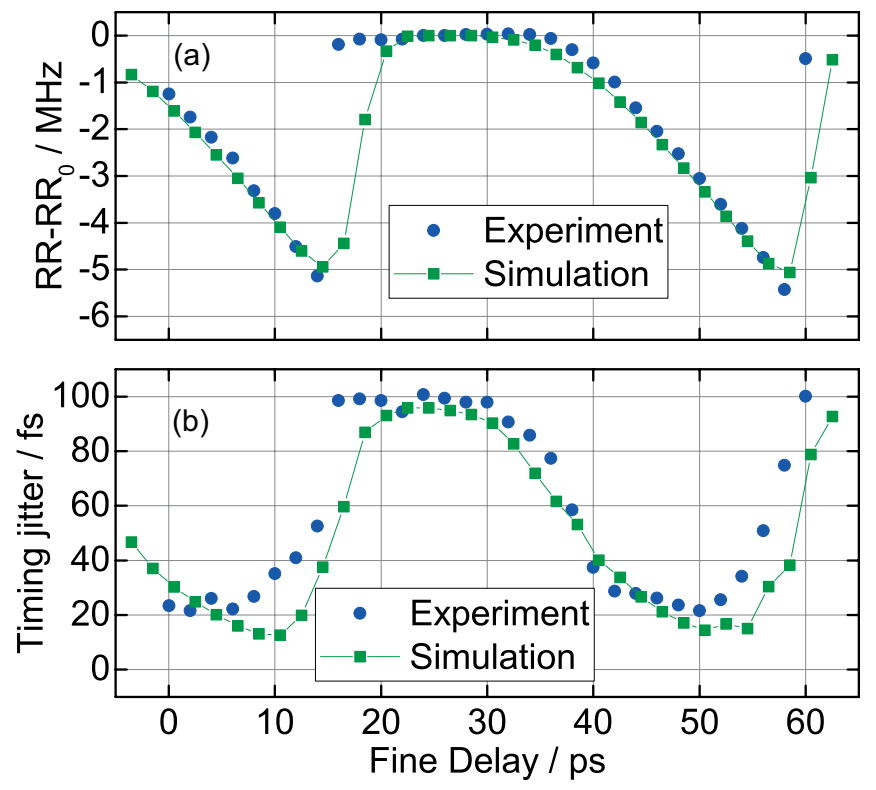

Figure 3. (a) Repetition rate (RR) deviation and (b) timing jitter in dependence on the optical feedback fine-delay for an optical feedback cavity length of $16 \mathrm{~m}$ : Experiment (blue) and simulation (green). 
The timing jitter dependence on the fine-delay exemplifies the same periodicity of $43 \mathrm{ps}$. Within one period two plateaus are observed. The first plateau ( $16 \mathrm{ps}$ to $30 \mathrm{ps}$ ) coincides with the plateau in repetition rate deviation and there the timing jitter of the laser subject to optical self-injection equals the solitary timing jitter. This underlines the absence of interaction between the back-injected pulses and the pulses within the laser cavity. For increasing fine delays the timing jitter decreases to the second plateau ( $42 \mathrm{ps}$ to $52 \mathrm{ps}$ ) within less than $10 \mathrm{ps}$ of additional delay. The timing jitter obtained in this second plateau amounts to values between $20 \mathrm{fs}$ and $30 \mathrm{fs}$ and the plateau spans over $10 \mathrm{ps}$ of additional time delay. In contrast to the transition from the lowest to the solitary repetition rate at the end of the period within a 2 ps time delay increase, the timing jitter continuously increases to the solitary value by $9.4 \mathrm{fs} / \mathrm{ps}$. While the dual-plateau shaped timing jitter characteristic perfectly resembles the results obtained for a straight waveguide passively mode-locked quantum-well laser, ${ }^{24}$ the increasing timing jitter at the end of the period differs significantly. This could indicate, that the back-injected pulses interact with the pulses propagating in the counter-clockwise direction within the cavity and stabilize this pulse train. By the symmetric dual absorber cavity design the counter-clockwise propagating pulses meet the clockwise propagating pulses within the absorbers in a colliding pulse mode-locking fashion and thereby improve the timing jitter of the clockwise propagating pulse train. At the end of the period, where the back-injected and the intracavity pulses nearly do not overlap, this indirect interaction could lessen the timing stability improvement. The best timing jitter amounts to $20 \mathrm{fs}$ and is obtained for a fine delay of $50 \mathrm{ps}$. The corresponding experimental TPN PSD of the repetition rate line is depicted in Fig. 4. The expected $\mathrm{f}^{2}$-dependency of the TPN PSD on the offset frequency is observed and a repetition rate line width of $38 \mathrm{kHz}$ can be determined and is indicated by the red line. The plateau for offset frequencies exceeding $2 \mathrm{MHz}$ at $-90 \mathrm{dBc} / \mathrm{Hz}$ to $-92 \mathrm{dBc} / \mathrm{Hz}$ stems from the noise of the measurement equipment. The peak at $12.51 \mathrm{MHz}$ and its harmonic is a noise-induced sideband and stems from the single-cavity optical self-injection. It has been shown in experiment and by simulations, that dual-cavity self-injection can suppress these sidebands. ${ }^{14,20,21}$ The corresponding simulation results for the

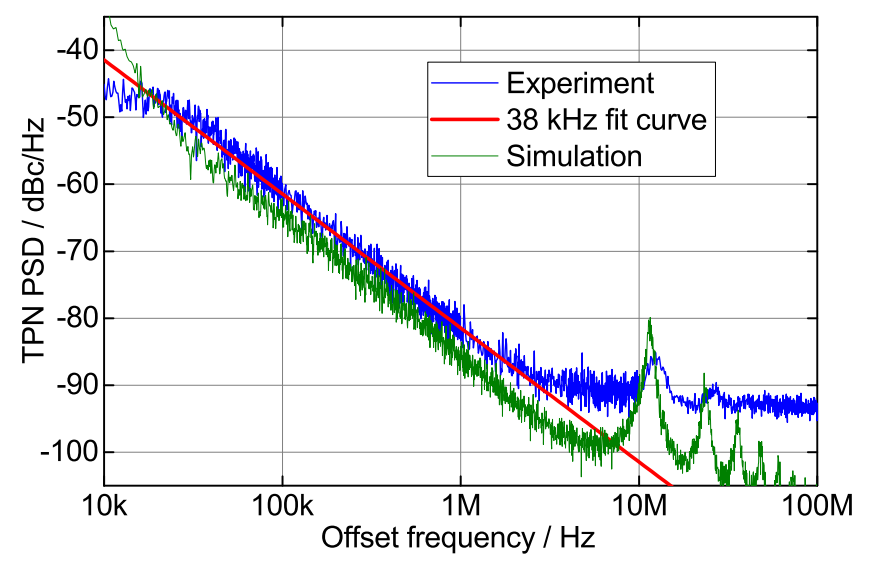

Figure 4. TPN PSD for the lowest experimentally achieved timing jitter value (blue) at a fine-delay of $50 \mathrm{ps}, \mathrm{f}^{2}$-fit curve indicating a line width of $38 \mathrm{kHz}$ (red) and simulated TPN PSD (green).

repetition rate deviations and for the timing jitter as functions of fine delay are depicted in Fig. 3(a) and (b), respectively, in green color. The simulation parameters used, obtained from the solitary experimental results and the cavity geometry are given by $\gamma=0.07, W(\Delta T)=3.1 \mathrm{ps}, \nu_{\text {ext }}=12.5 \mathrm{MHz}$, and $\sigma_{\mathrm{ptp}}=99 \mathrm{fs}$. The simulated repetition rate deviation with increasing fine delay reproduces the experimental findings quantitatively in good agreement. The plateau-structure, stemming from the limited interaction width, the periodicity in fine delay, corresponding to the internal laser roundtrip time, and the decreasing slope are reproduced. For the timing jitter, the periodicity in fine delay and the dual-plateau shape of the data is also reproduced qualitatively and quantitatively. However, the simulations do not reproduce the timing jitter increase with increasing fine delay at the end of the period. Since this increase seems to originate from the dual interaction between back-injected pulses and counter-clockwise propagating pulses and counter-clockwise propagating pulses and clockwise propagating pulses within the symmetric ring cavity, and the model is capable of simulating the interaction of back-injected and emitted pulse, more research in this direction is envisioned. The simulated TPN PSD spectrum for a fine delay of 50 ps is depicted in Fig. 4 in green color. The lowest timing jitter is obtained in the simulations, is 
depicted. The slope corresponds well to the experimental TPN PSD spectrum, showing the excellent agreement between simulation and experiment. The sidebands are much more pronounced in the simulations than in the experiments. The smaller sidebands in the experiments can stem from the fact that the clockwise propagating pulses are interacting with the optical seld-injected counterclockwise propagating pulses only in the absorber section and not in the whole device as in straight waveguide lasers producing higher sidebands compared to this ring structure.

\section{CONCLUSION}

In conclusion, optical self-injection stabilization on an InP photonic integrated circuit extended cavity passively mode-locked ring laser has been demonstrated. Repetition rate tuning up to $5.5 \mathrm{MHz}$ and a timing jitter reduction by optical self-injection from $99 \mathrm{fs}$, in solitary laser operation, to $20 \mathrm{fs}$ has been obtained. Experimental results are in excellent agreement with results from a stochastic model originally developed to study optical self-injection of edge-emitting straight waveguide semiconductor lasers, thus showing that the ring structure subject to optical self-injection exhibits the same behaviour in timing jitter reduction and repetition rate tunability as FabryPerot lasers featuring the same cavity roundtrip time, indicating a similar intra-cavity pulse interaction of the back-injected pulses with the original pulses.

\section{ACKNOWLEDGMENTS}

Dominik Auth and Stefan Breuer acknowledge support by the Dr. Hans Messer Foundation (Doctoral scholarship); Authors thank W. Elsäßer for support; Stefan Breuer acknowledges support by the German Research Foundation (DFG) (389193326).

\section{REFERENCES}

[1] Van Gasse, K., Uvin, S., Moskalenko, V., Latkowski, S., Roelkens, G., Bente, E., and Kuyken, B., "Recent advances in the photonic integration of mode-locked laser diodes," IEEE Photonics Technol. Lett. , 1-1 (2019).

[2] Vujicic, V., Calò, C., Watts, R., Lelarge, F., Browning, C., Merghem, K., Martinez, A., Ramdane, A., and Barry, L. P., "Quantum dash mode-locked lasers for data centre applications," IEEE J. Sel. Top. Quantum Electron. 21, 53-60 (Nov 2015).

[3] Picqué, N. and Haensch, T., "Frequency comb spectroscopy," Nat. Photonics 13, 146-157 (02 2019).

[4] Latkowski, S., Moskalenko, V., Tahvili, S., Augustin, L., Smit, M., Williams, K., and Bente, E., "Monolithically integrated $2.5 \mathrm{ghz}$ extended cavity mode-locked ring laser with intracavity phase modulators," Opt. Lett. 40, 77-80 (Jan 2015).

[5] Hei, K., Shi, G., Hänsel, A., Deng, Z., Latkowski, S., Van den Berg, S. A., Bente, E., and Bhattacharya, N., "Distance metrology with integrated mode-locked ring laser," IEEE Photonics J., , 1-1 (2019).

[6] Moskalenko, V., Koelemeij, J., Williams, K., and Bente, E., "Study of extra wide coherent optical combs generated by a QW-based integrated passively mode-locked ring laser," Opt. Lett. 42, 1428-1431 (Apr 2017).

[7] Keyvaninia, S., Uvin, S., Tassaert, M., Fu, X., Latkowski, S., Mariën, J., Thomassen, L., Lelarge, F., Duan, G., Verheyen, P., Lepage, G., Campenhout, J. V., Bente, E., and Roelkens, G., "Narrow-linewidth short-pulse iii-v-on-silicon mode-locked lasers based on a linear and ring cavity geometry," Opt. Express 23, 3221-3229 (Feb 2015).

[8] Lo, M.-C., Auth, D., Weber, C., Fiala, P., Sauer, P., Carpintero, G., and Breuer, S., "Photonic integrated circuit extended cavity passively mode-locked dual absorber symmetric ring laser," Opt. Lett. 44, 3566-3569 (Jul 2019).

[9] Moskalenko, V., Latkowski, S., Tahvili, S., de Vries, T., Smit, M., and Bente, E., "Record bandwidth and sub-picosecond pulses from a monolithically integrated mode-locked quantum well ring laser," Opt. Express 22(23), 28865-28874 (2014).

[10] Nielsen, L., Bente, E. A. J. M., den Haan, E., and Heck, M. J. R., "Theoretical and experimental investigation of unidirectionality in an integrated semiconductor ring mode-locked laser with two saturable absorbers," IEEE J. Quantum Electron. 54, 1-10 (Oct 2018). 
[11] Chen, Y.-K. and Wu, M. C., "Monolithic colliding-pulse mode-locked quantum-well lasers," IEEE J. Quantum Electron. 28(10), 2176-2185 (1992).

[12] Solgaard, O. and Lau, K. Y., "Optical feedback stabilization of the intensity oscillations in ultrahighfrequency passively modelocked monolithic quantum-well lasers," IEEE Photonics Technol. Lett. 5, 12641267 (Nov 1993).

[13] Rauch, S., Drzewietzki, L., Klehr, A., Sacher, J., Elsäßer, W., and Breuer, S., "Experimental study of the timing jitter of a passively mode-locked external-cavity semiconductor laser subject to repetition rate transitions and optical feedback," IEEE J. Quantum Electron. 51, 1-7 (April 2015).

[14] Haji, M., Hou, L., Kelly, A. E., Akbar, J., Marsh, J. H., Arnold, J. M., and Ironside, C. N., "High frequency optoelectronic oscillators based on the optical feedback of semiconductor mode-locked laser diodes," Opt. Express 20, 3268-3274 (Jan 2012).

[15] Rosales, R., Merghem, K., Martinez, A., Akrout, A., Tourrenc, J. ., Accard, A., Lelarge, F., and Ramdane, A., "Inas/inp quantum-dot passively mode-locked lasers for 1.55- $\mu \mathrm{m}$ applications," IEEE J. Sel. Top. Quantum Electron. 17, 1292-1301 (Sep. 2011).

[16] Weber, C., Batagelj, B., Fiala, P., Auth, D., Dragonja, U., Gaimard, Q., Ramdane, A., and Breuer, S., "Quantum dash frequency comb laser stabilisation by optical self-injection provided by an all-fibre based delay-controlled passive external cavity," Electron. Lett. 55(18), 1006-1009 (2019).

[17] Arsenijević, D., Kleinert, M., and Bimberg, D., "Phase noise and jitter reduction by optical feedback on passively mode-locked quantum-dot lasers," Appl. Phys. Lett. 103(23), 231101 (2013).

[18] Lin, C., Grillot, F., Li, Y., Raghunathan, R., and Lester, L. F., "Microwave characterization and stabilization of timing jitter in a quantum-dot passively mode-locked laser via external optical feedback," IEEE J. Sel. Top. Quantum Electron. 17, 1311-1317 (Sep. 2011).

[19] Drzewietzki, L., Breuer, S., and Elsäßer, W., "Timing jitter reduction of passively mode-locked semiconductor lasers by self- and external-injection: Numerical description and experiments," Opt. Express 21, 16142-16161 (Jul 2013).

[20] Nikiforov, O., Jaurigue, L., Drzewietzki, L., Lüdge, K., and Breuer, S., "Experimental demonstration of change of dynamical properties of a passively mode-locked semiconductor laser subject to dual optical feedback by dual full delay-range tuning," Opt. Express 24, 14301-14310 (Jun 2016).

[21] Jaurigue, L., Nikiforov, O., Schöll, E., Breuer, S., and Lüdge, K., "Dynamics of a passively mode-locked semiconductor laser subject to dual-cavity optical feedback," Phys. Rev. E 93, 022205 (Feb 2016).

[22] Breuer, S., Elsäßer, W., McInerney, J. G., Yvind, K., Pozo, J., Bente, E. A. J. M., Yousefi, M., Villafranca, A., Vogiatzis, N., and Rorison, J., "Investigations of repetition rate stability of a mode-locked quantum dot semiconductor laser in an auxiliary optical fiber cavity," IEEE J. Quantum Electron. 46, 150-157 (Feb 2010).

[23] Stutz, S., Auth, D., Weber, C., Drzewietzki, L., Nikiforov, O., Rosales, R., Walther, T., Lester, L. F., and Breuer, S., "Dynamic intermode beat frequency control of an optical frequency comb single section quantum dot laser by dual-cavity optical self-injection," IEEE Photonics J. 11, 1-8 (Oct 2019).

[24] Auth, D., Drzewietzki, L., Weber, C., Klehr, A., Knigge, A., and Breuer, S., "Repetition rate control of optical self-injected passively mode-locked quantum-well lasers: experiment and simulation," Electron. Lett. 54, 374-376 (March 2018).

[25] Williams, K., Bente, E., Heiss, D., Jiao, Y., Ławniczuk, K., Leijtens, X., van der Tol, J., and Smit, M., "InP photonic circuits using generic integration," Photonics Res. 3(5), B60-B68 (2015).

[26] Kéfélian, F., O’Donoghue, S., Todaro, M. T., McInerney, J. G., and Huyet, G., "RF linewidth in monolithic passively mode-locked semiconductor laser," IEEE Photonics Technol. Lett. 20(16), 1405-1407 (2008). 